\title{
Patterns of Relapse in Squamous Cell Carcinoma of the Tonsil - Unilateral vs. Bilateral Radiation in the HPV-Era
}

\author{
Allison Ye ${ }^{1}$, Katherine L. Bradley ${ }^{2}$, Hosam Kader ${ }^{1}$, John $\mathrm{Wu}^{1}$, John H. Hay ${ }^{1}$ \\ 1. Department of Radiation Oncology, British Columbia Cancer Agency - Vancouver Cancer Centre 2. \\ Department of Oncology, Queen Alexandra Hospital
}

$\square$ Corresponding author: Allison Ye, allison.ye@bccancer.bc.ca

Disclosures can be found in Additional Information at the end of the article

\section{Abstract}

Objectives: In the pre-human papillomavirus (HPV) era, unilateral radiation therapy (URT) for tonsil cancer was associated with low contralateral failure rates and had less toxicity than bilateral radiation therapy (BRT). This study explores the validity of URT in HPV-positive tonsil cancers.

Methods: Tonsil squamous cell carcinomas (SCC) treated (typically with 70 Gy radiation and Cisplatin-based chemotherapy) between 2001 and 2007 were reviewed. Retrospective p16 immunohistochemistry staining was undertaken. Baseline, treatment, and response data were collected.

Results: Of 182 patients, 78\% were p16-positive, were younger (predominantly male), mostly former or non-smokers, and had a more advanced nodal stage. With a median follow-up of 68 months, contralateral recurrence (CLR) rates were low (3.5\% p16-positive versus 2.5\% p16negative, $\mathrm{p}=0.63$ ). Overall survival (OS) was $74 \%$ for $\mathrm{p} 16$-positive versus $54 \%$ for $\mathrm{p} 16$-negative subjects $(\mathrm{p}=0.01)$, but all other outcomes were similar. Analysis amongst only p16-positive subjects revealed URT was delivered to $37 \%$, with CLR rates of $7.5 \%$ versus $1.1 \%$ for those treated with BRT, $\mathrm{p}=0.05$. Of the four p16-positive subjects treated with URT who developed contralateral recurrences, three were managed with neck dissection (two disease-free and one died of lung metastases) and one received palliative radiation to the neck and distant metastatic site. All disease control and survival outcomes were similar between those treated with URT versus BRT.

Conclusion: While CLRs remain rare overall, there appears to be a slightly increased rate among HPV-positive subjects treated with URT. However, overall outcomes do not appear to be

Received 05/02/2015

Review began 05/04/2015

Review ended 08/31/2015

Published 09/10/2015

C Copyright 2015

Ye et al. This is an open access article distributed under the terms of the Creative Commons Attribution License CC-BY 3.0., which permits unrestricted use, distribution, and reproduction in any medium, provided the original author and source are credited. impacted, suggesting that URT remains a reasonable approach in HPV-positive subjects.

Categories: Otolaryngology, Radiation Oncology, Oncology

Keywords: tonsil cancer, squamous cell carcinoma, head and neck, radiation, unilateral, bilateral, hpv, recurrence, p16, human papillomavirus

\section{Introduction}

There is known recognition of the role of human papillomavirus (HPV) in the etiology of head and neck cancers, notably those arising from the tonsil, base of tongue or elsewhere in the oropharynx [1]. Age-adjusted incidence of cancers of the oral cavity and larynx have declined 
along with tobacco usage. In contrast, oropharyngeal cancer incidence has increased, particularly in men, over a similar time period [2-3]. Increasing numbers of oropharyngeal cases are likely to be HPV-positive, although testing for HPV, or a surrogate, was not routinely conducted during the study period. Compared with HPV-negative oropharyngeal cancers, HPVpositive patients tend to be younger and use less alcohol and tobacco. HPV-positive cancers tend to present with a small, or occult, primary tumour, but frequently with large nodal involvement [4-5]. As a group, they also have a relatively favourable response to therapy with improved locoregional tumour control, improved disease-specific survival, and improved overall survival following radiotherapy (RT) or surgery when compared to HPV-negative cancers [6-9].

The use of unilateral RT (URT) techniques (also commonly known as ipsilateral RT) to treat patients with carcinoma of the tonsil reduces acute and late toxicity relative to bilateral techniques. In addition to producing less acute mucositis, URT reduces long-term damage to the salivary glands [10-12]. Sparing of one submandibular gland is beneficial, as these glands produce most of the resting saliva [13]. URT also reduces the dose to the contralateral carotid artery, potentially reducing the risk of stroke [14]. Finally, it has also been shown to reduce long-term dysphagia [12]. In the pre-HPV-era literature (namely, before the 2000s when smoking was the main driver of head and neck cancers), based on retrospective series comparisons, ipsilateral techniques gave results at least as good as those reported with bilateral techniques, with a low risk of failure in the contralateral neck [15-16]. There is no definitive evidence of increase in the risk of contralateral lymph node positivity in the HPV-era [17]; however, given the propensity of HPV-positive oropharyngeal cancer to spread at an early stage to the lymph nodes, there is a potential for increased risk associated with unilateral radiation. The purpose of this project is to explore the validity of URT in the HPV-era.

\section{Materials And Methods}

Records for all patients referred to the British Columbia Cancer Agency (BCCA) with squamous cell carcinoma (SCC) of the tonsil between January 1, 2001 and December 31, 2007 were retrieved from the provincial database. Search parameters were ICD codes C10.2 (lateral wall), C09.9 (tonsil), C09.0 (tonsillar fossa), and C09.1 (tonsillar pillars). Those who were of nonsquamous histology, incorrectly classified with respect to tumour site, or not treated at BCCA were excluded. Electronic narrative patient charts were used to gather data on patient and treatment characteristics as well as outcomes. RT treatment plans were used to confirm treatment volumes where narrative charts were incomplete.

HPV status was determined through retrospective p16 immunohistochemistry (IHC) staining, given the recognised correlation between HPV DNA and p16 protein expression $[5,18]$. Testing was carried out on available tissue samples, selecting those with the highest likelihood of having adequate tissue content. Four micrometre sections of formalin-fixed paraffin embedded tissues were analysed using a tissue microarray approach. $\mathrm{p} 16^{\mathrm{INK} 4 \mathrm{a}} \mathrm{IHC}$ was determined using mouse monoclonal primary antibodies from the CINtec(R) Cytology Kit (Roche mtm laboratories AG, Heidelberg, Germany). Those with IHC staining above $30 \%$ of cells were considered to be positive.

Staging investigations routinely consisted of flexible endoscopy, biopsy, CT scan of the neck, and chest $\mathrm{x}$-ray. Examination under anesthesia (EUA) was only undertaken when a primary site was not easily indentified. After 2005, PET scans began to be used, but very infrequently. Less than $10 \%$ of patients in the study underwent either a diagnostic or routine follow-up PET scan.

Patients were CT simulated in the supine position with mask fixation. The usage of bite blocks was not uniform and varied by the regional centre. Gross tumour volumes were contoured using 
the CT simulation scan, and PET scan where available. A 5-7 mm margin was added for clinical target volumes and a 5-7 mm margin for planning target volumes. Subjects were treated with 66-70 Gy in 2 Gy daily fractions (46\%), 60 Gy in 2.4 Gy daily fractions (42\%), or more rarely, 55 Gy in 2.75 Gy daily fractions (7\%) or 66 Gy in 2 Gy fractions concomitant boost (6\%). These dose levels were to gross disease, with elective nodal volumes treated to 50-60 Gy. With respect to RT techniques, treatment was delivered with 3D conformal radiation therapy (3DCRT) or in the more modern era, intensity-modulated radiation therapy (IMRT). Dose and fractionation schedules, as well as the decision to use either ipsilateral or bilateral irradiation, was at the discretion of the treating oncologist. However, primary tumours with a significant extension on to the soft palate or base of tongue, or extending to within $1 \mathrm{~cm}$ of the midline were given bilateral treatment, as were those with bilateral lymph node disease. In unilateral treatments, the dose to the contralateral submandibular and parotid glands was $<20 \mathrm{~Gy}$ whether treated with 3DCRT or IMRT.

Concomitant chemotherapy regimens were either Cisplatin $100 \mathrm{mg} / \mathrm{m}^{2}$ on Days 1,22 , and 43, or Carboplatin 70 mg/m², along with 5-FU 600 mg/m² on Days 1-4, 22-25, and 43-46. Carboplatin was used for subjects with renal impairment who could not receive Cisplatin.

All outcomes were measured from the date of diagnosis. Time to local, regional (ipsilateral or contralateral), or distant failure, death from disease or another cause, or last follow-up was recorded. Those with persistent disease treated with curative intent salvage treatment were not recorded as relapses unless they later had a separate failure. Those whose persistent disease was treated with palliative intent were defined as locoregional failures at the end of the treatment date. Disease-free survival (DFS) was defined as time to local, regional, or distant failure while disease-specific survival (DSS) was defined as time to death from tonsil SCC. Outcomes were censored at the date of the last contact.

All analysis was completed using Statistical Package for Social Sciences version 14.0. In addition to descriptive analysis, independent sample t-tests and Pearson Chi-square cross tabulations were used to make comparisons between both p16-positive and negative subjects, as well as those treated with unilateral versus bilateral RT. Actuarial outcomes analysis was completed using Kaplan-Meier and log-rank analysis.

This study was approved by the British Columbia Cancer Agency Institutional Research Ethics Board (approval \#H11-00364). Informed patient consent had been obtained at the time of treatment.

\section{Results}

A total of 405 cases of tonsil cancer between 2001 and 2007 were retrieved from the provincial database. Eighty-three patients were excluded due to classification errors or not receiving treatment at the agency. One hundred and forty patients were excluded because p16 status could not be determined - either due to an inability to procure the pathology sample or insufficient tissue to allow testing. A total of 182 charts were then reviewed.

\section{p16-positive versus p16-negative}

Of these, 142 patients (78\%) were p16-positive. These subjects tended to be younger, male, former or non-smokers, and had more advanced nodal and overall stage. RT, chemotherapy, and surgery treatment characteristics were similar (Table 1). Rates of contralateral recurrence (CLR) were low overall and did not differ by p16 status (3.5\% p16-positive versus $2.5 \%$ p16-negative, $\mathrm{p}=0.75)$. With a median follow-up of 68 months for all patients (73.5 months for $\mathrm{p} 16-$ positive and 53.5 months for p16-negative), local control (LC), locoregional control (LRC), DFS, 
Cureus

and DSS were statistically similar, but the five-year overall survival (OS) was $74 \%$ for p16positive versus $54 \%$ for $\mathrm{p} 16$-negative subjects $(\mathrm{p}=0.01)$ (Table 2 ).

\begin{tabular}{|c|c|c|c|}
\hline Characteristic & p16-positive (n=142) & p16-negative $(n=40)$ & $\mathrm{p}$-value \\
\hline Mean age at diagnosis & 55.7 & 59.5 & 0.02 \\
\hline Gender & & & $<0.01$ \\
\hline Male & $117(82 \%)$ & $23(58 \%)$ & \\
\hline Female & $25(18 \%)$ & 17 (43\%) & \\
\hline T Stage & & & 0.37 \\
\hline T1 & 44 (31\%) & $8(20 \%)$ & \\
\hline T2 & $67(47 \%)$ & 19 (48\%) & \\
\hline T3 & $24(17 \%)$ & $11(28 \%)$ & \\
\hline T4 & $7(5 \%)$ & $2(5 \%)$ & \\
\hline N Stage & & & $<0.01$ \\
\hline No & $26(18 \%)$ & $18(45 \%)$ & \\
\hline N1 & $26(18 \%)$ & $5(13 \%)$ & \\
\hline N2 & 75 (53\%) & 15 (38\%) & \\
\hline N3 & $15(11 \%)$ & $2(5 \%)$ & \\
\hline Stage & & & 0.03 \\
\hline I & $3(2 \%)$ & $4(10 \%)$ & \\
\hline II & $18(13 \%)$ & $9(23 \%)$ & \\
\hline III & $28(20 \%)$ & $10(25 \%)$ & \\
\hline IVA & $78(55 \%)$ & $15(38 \%)$ & \\
\hline IVB & $15(11 \%)$ & $2(5 \%)$ & \\
\hline Smoking & & & $<0.01$ \\
\hline Current/quit less than 1 year & $42(30 \%)$ & $31(78 \%)$ & \\
\hline Former (quit more than 1 year) & 55 (39\%) & $2(5 \%)$ & \\
\hline Never & $45(32 \%)$ & $7(18 \%)$ & \\
\hline \multicolumn{4}{|l|}{ Radiation } \\
\hline$\leq 60$ Gy & $66(46 \%)$ & $21(53 \%)$ & \multirow{3}{*}{0.5} \\
\hline 61-70 Gy & $76(54 \%)$ & $19(48 \%)$ & \\
\hline Unilatera & $53(37 \%)$ & $17(43 \%)$ & \\
\hline
\end{tabular}




\section{Cureus}

$\begin{array}{lll}\text { Bilateral } & 89(63 \%) & 23(58 \%) \\ \text { RT alone } & 83(59 \%) & 29(73 \%) \\ \text { CRT } & 59(42 \%) & 11(28 \%) \\ \text { Chemotherapy } & & 8(73 \%) \\ \text { Cisplatin-based } & 47(80 \%) & 2(27 \%) \\ \text { Carboplatin-based } & 12(20 \%) & \\ \text { Surgery } & & 39(98 \%) \\ \text { None } & 128(90 \%) & 0 \\ \text { Primary resection } & 1(<1 \%) & 0 \\ \text { Post-RT primary and UND } & 1(<1 \%) & 1(3 \%) \\ \text { Post-RT UND } & 12(9 \%) & \end{array}$

\section{TABLE 1: Baseline patient and treatment characteristics, by p16 status}

$\mathrm{CRT}=$ Chemoradiation, $\mathrm{RT}=$ Radiation, UND = unilateral neck dissection

\begin{tabular}{|c|c|c|c|c|c|c|}
\hline & \multicolumn{3}{|l|}{ p16 status } & \multicolumn{3}{|c|}{ Laterality of Radiation } \\
\hline & p16-positive & p16-negative & Log rank p-value & URT & BRT & Log rank p-value \\
\hline LC & $90 \%$ & $83 \%$ & 0.24 & $89 \%$ & $87 \%$ & 0.69 \\
\hline LRC & $84 \%$ & $79 \%$ & 0.34 & $83 \%$ & $83 \%$ & 0.94 \\
\hline DFS & $78 \%$ & $74 \%$ & 0.43 & $78 \%$ & $78 \%$ & 0.99 \\
\hline DSS & $82 \%$ & $76 \%$ & 0.36 & $83 \%$ & $79 \%$ & 0.26 \\
\hline OS & $74 \%$ & $54 \%$ & 0.01 & $72 \%$ & $68 \%$ & 0.34 \\
\hline
\end{tabular}

TABLE 2: Five year outcomes by $\mathrm{p} 16$ status and laterality of radiation

\section{Unilateral versus bilateral radiation}

Overall, 70 subjects (38\%) were treated with URT. URT treated patients were similar in gender and age, but of earlier $\mathrm{T}, \mathrm{N}$, and overall stage, as compared to those who received BRT. In addition, the total dose of RT was more likely to be less than or equal to $60 \mathrm{~Gy}$ in those receiving URT. While those who received URT were more likely to have CLR (7.1\%) than those treated with bilateral RT (BRT) $(0.9 \%)(p=0.02)$, the LC, LRC, DFS, DSS, and OS were not worse for URT subjects (Table 2). Amongst URT subjects only, both crude CLR (7.5\% p16-positive and 5.9\% 


\section{Cureus}

p16-negative, $\mathrm{p}=0.82$ ) and actuarial CLR did not differ by p16 status (Figure 1).

URT only

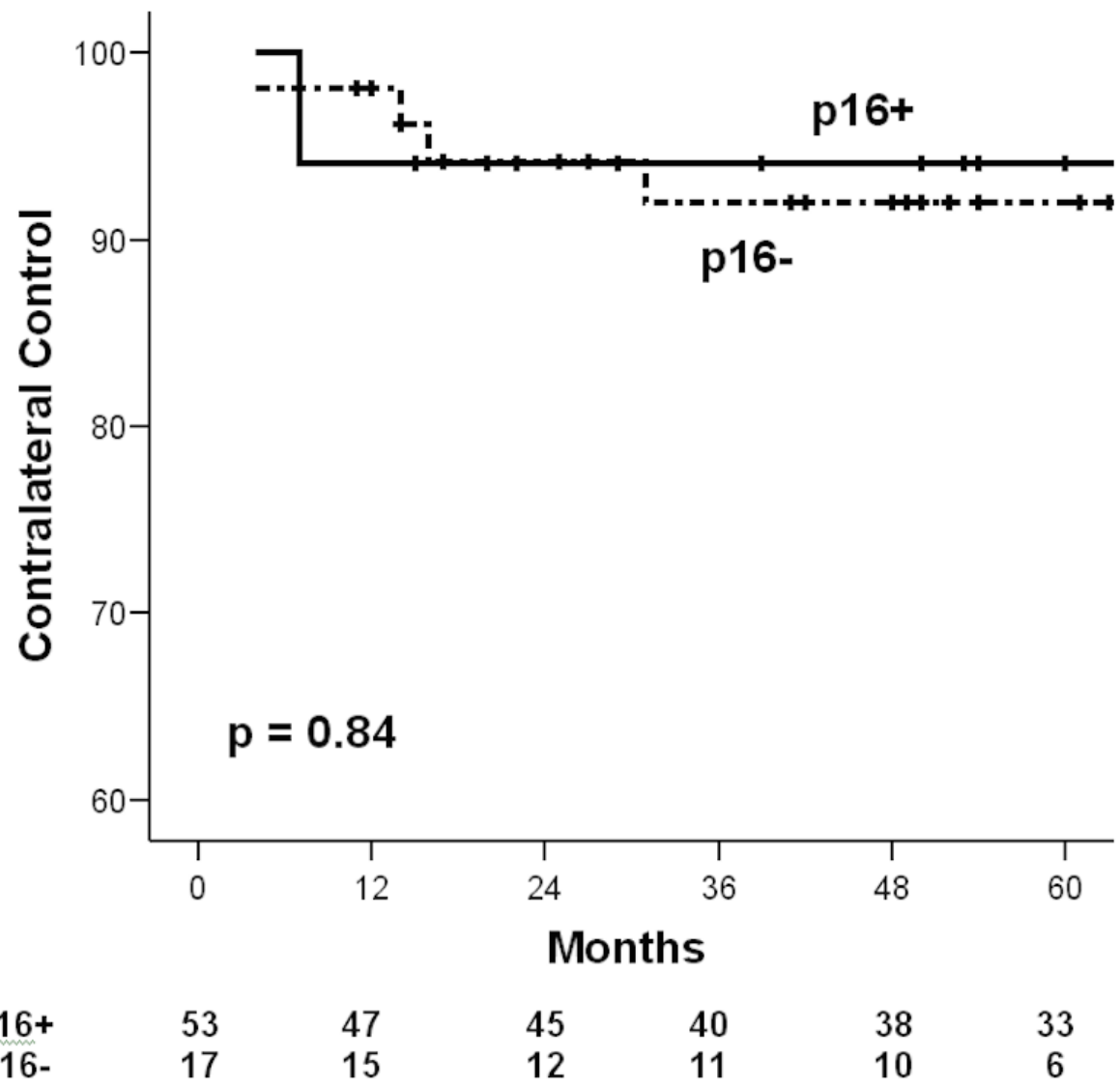

Number of patients at risk

FIGURE 1: Contralateral recurrences for URT subjects, by $p 16$ status

\section{p16-positive only, unilateral vs. bilateral radiation}

Among the 142 p16-positive subjects, 53 were treated with unilateral RT (URT). Those treated with URT versus BRT were similar in age and gender, but those treated with URT were of less advanced nodal and overall stage. Compared with those who received BRT, URT subjects were again more likely to have been treated with RT doses less than or equal to $60 \mathrm{~Gy}$. Those treated with URT were more likely to be treated with RT alone, but the choice of the chemotherapeutic regimen was similar (Table 3).

Characteristic

Mean age at diagnosis

Gender
Unilateral RT ( $n=53)$

55.2
Bilateral RT ( $\mathbf{n = 8 9 )}$

56.0 p-value

0.65

0.09 


\section{Cureus}

Male

Female

T Stage

T1

T2

T3

T4

N Stage

NO

N1

$\mathrm{N} 2 \mathrm{a}$

$\mathrm{N} 2 \mathrm{~b}$

N2c

N3

\section{Stage}

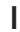

II

III

IVA

IVB

Smoking

Current/quit less than 1 year

Former (quit more than 1 year)

Never

Radiation

$\leq 60$ Gy

61-70 Gy

RT alone

CRT

Chemotherapy

Cisplatin-based
$43(81 \%)$

$10(19 \%)$

$22(42 \%)$

$28(53 \%)$

$3(6 \%)$

0

$15(28 \%)$

$18(34 \%)$

7 (13\%)

$11(21 \%)$

0

$2(4 \%)$

$1(2 \%)$

$13(25 \%)$

19 (36\%)

$18(34 \%)$

$2(4 \%)$

$13(25 \%)$

$28(53 \%)$

$12(23 \%)$

$35(66 \%)$

18 (34\%)

42 (79\%)

$11(21 \%)$

19 (91\%)
74 (83\%)

15 (17\%)

$<0.01$

$22(25 \%)$

$39(44 \%)$

$21(24 \%)$

$8(8 \%)$

$<0.01$

$11(12 \%)$

$8(9 \%)$

$9(10 \%)$

$36(40 \%)$

$12(14 \%)$

13 (15\%)

$<0.01$

$2(2 \%)$

$5(6 \%)$

$9(10 \%)$

$60(67 \%)$

$13(15 \%)$

0.03

$29(33 \%)$

27 (30\%)

$33(37 \%)$

31 (35\%)

58 (65\%)

$<0.01$

$41(46 \%)$

$<0.01$

48 (54\%)

0.30 


\section{Cureus}

Carboplatin-based

$1(9 \%)$

$11(23 \%)$

Surgery

None

Primary resection

Post-RT primary and UND

Post-RT UND
$51(96 \%)$

0

0

$2(4 \%)$
$77(87 \%)$

$1(1 \%)$

$1(1 \%)$

$10(11 \%)$

TABLE 3: Baseline characteristics of p16-positive subjects, by laterality of radiation

After completion of primary treatment, 81\% of p16-positive subjects had a complete clinical response at first assessment after completion of primary treatment. There were more complete responses in the URT group (93\% vs. 74\%, p < 0.01).

CLRs were seen in four (7.5\%) subjects treated with URT versus one (1.1\%) treated with BRT (Figure 2). Three of the four URT subjects with CLR were treated with a neck dissection while one received palliative RT for the CLR and concurrent pulmonary metastases (and died of his disease over one year later). Of the three patients who underwent neck dissection, one was disease-free at last contact, one died of pulmonary metastases, and one died of a second primary cancer of the lung. The subject treated with BRT underwent bilateral neck dissection but developed ipsilateral subcutaneous metastases within five months and died of their disease. 


\section{Cureus}

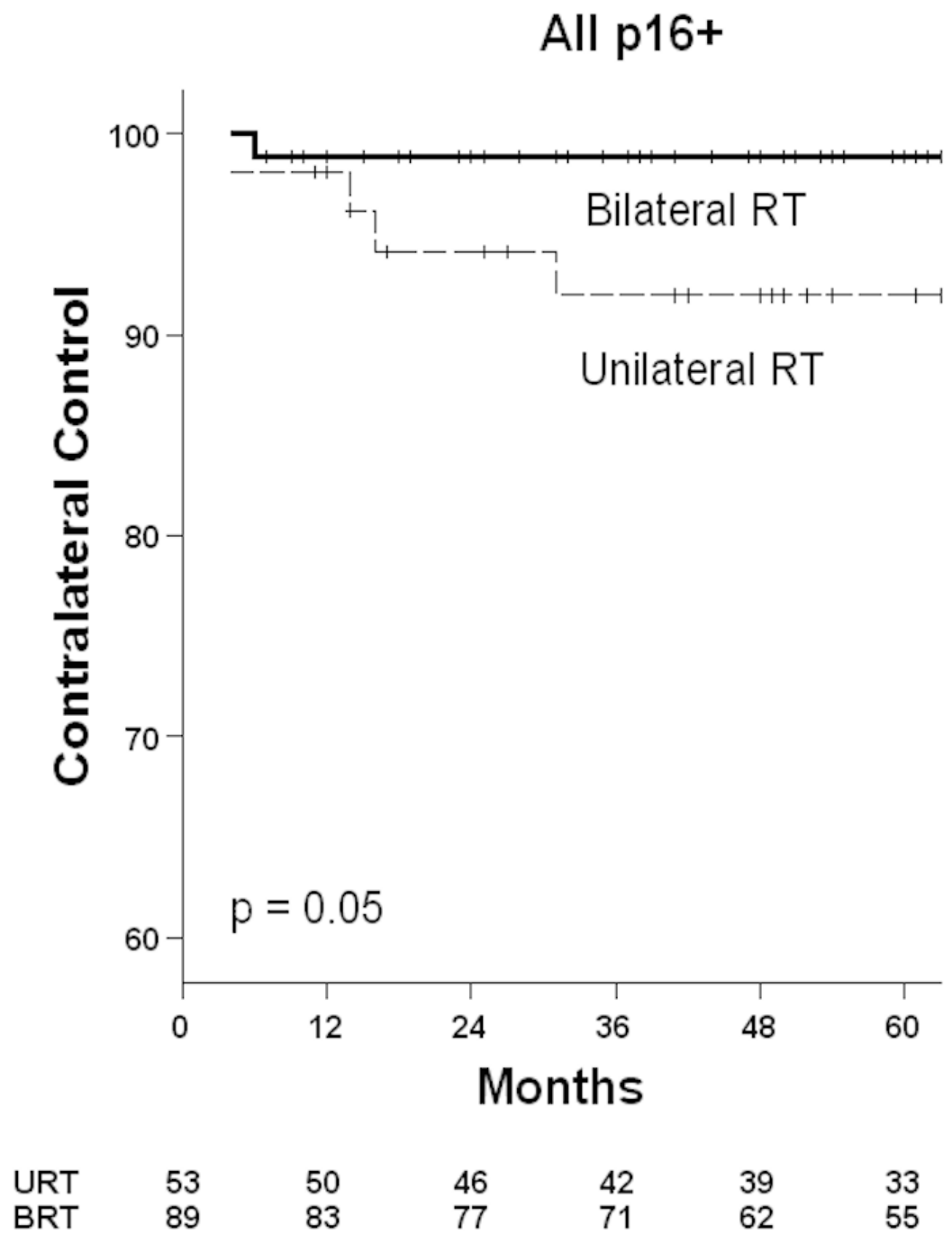

Number of patients at risk

FIGURE 2: Contralateral recurrences for p16-positive subjects, by laterality of RT

Among the p16-positive subjects treated with URT, CLR did not correlate with tumour, nodal, or overall stage. Certain subgroups had very small numbers limiting this analysis (Table 4). 


\section{Cureus}

\begin{tabular}{|c|c|c|c|}
\hline & $\mathbf{n}$ & CLR (\%) & $p$-value \\
\hline T1 & 22 & $1(5 \%)$ & \multirow{3}{*}{0.10} \\
\hline T2 & 28 & 2 (7\%) & \\
\hline T3 & 3 & $1(33 \%)$ & \\
\hline No & 15 & 0 & \multirow{6}{*}{0.26} \\
\hline N1 & 18 & $3(17 \%)$ & \\
\hline N2a & 7 & 1 (14\%) & \\
\hline N2b & 11 & 0 & \\
\hline N2c & 0 & 0 & \\
\hline N3 & 2 & 0 & \\
\hline Stage I & 1 & 0 & \multirow{5}{*}{0.45} \\
\hline Stage II & 13 & 0 & \\
\hline Stage III & 19 & 3 (16\%) & \\
\hline Stage IVA & 18 & $1(6 \%)$ & \\
\hline Stage IVB & 2 & 0 & \\
\hline
\end{tabular}

TABLE 4: Contralateral recurrences by $\mathrm{T}, \mathrm{N}$, and overall stage for $\mathrm{p} 16$-positive subjects treated with unilateral radiation therapy

LC, LRC, DSS, DFS, and OS curves are shown in Figure 3. Despite the difference in contralateral recurrences, no differences in overall outcomes were seen for those treated with URT versus BRT. 


\section{Cureus}
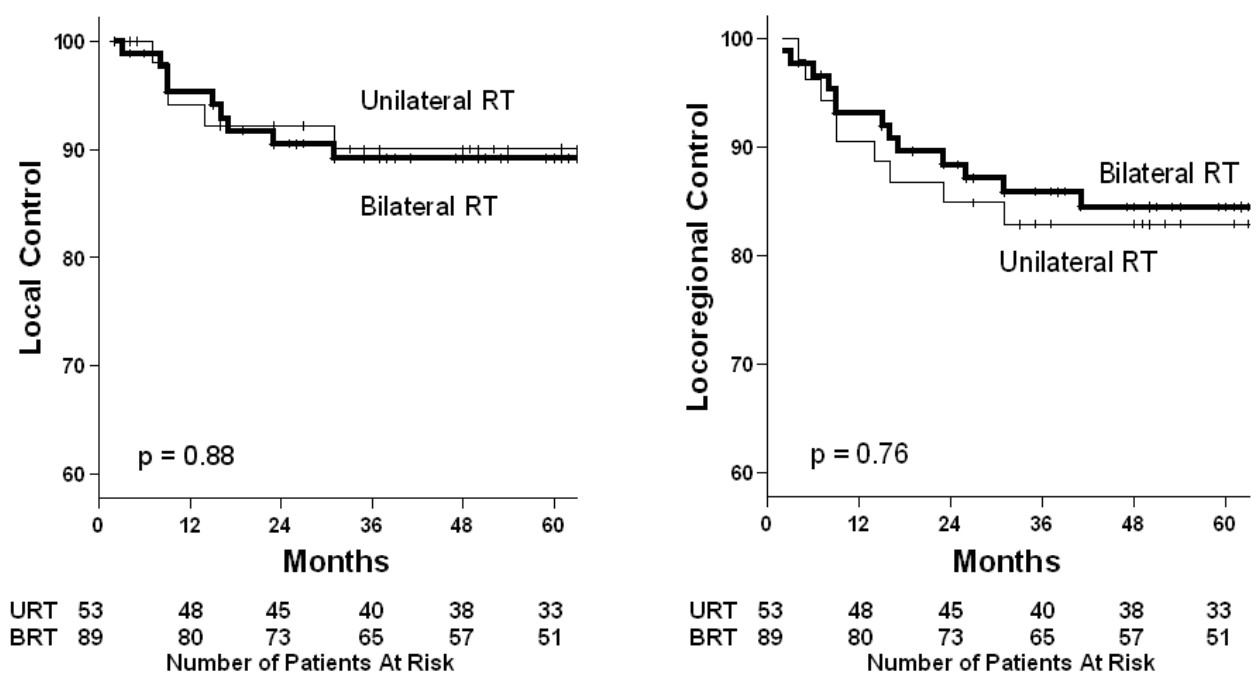

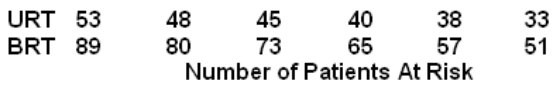
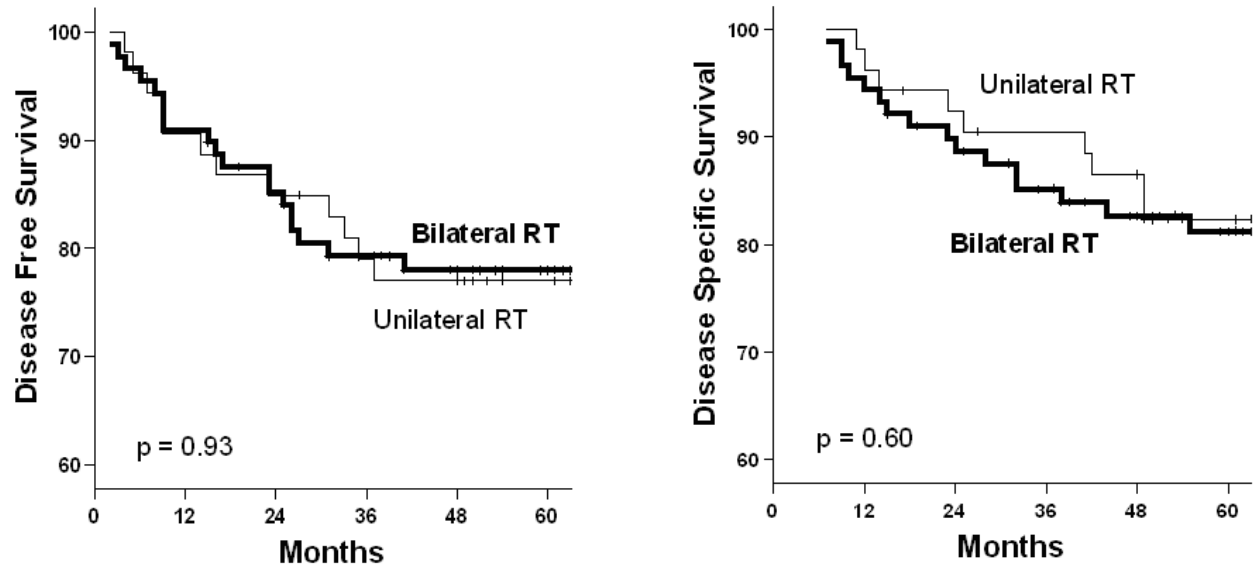

$\begin{array}{lllllll}\text { URT } & 53 & 48 & 45 & 40 & 38 & 33 \\ \text { BRT } & 89 & 80 & 73 & 65 & 57 & 51\end{array}$

Number of Patients At Risk

$\begin{array}{lllllll}\text { URT } & 53 & 51 & 48 & 45 & 42 & 35 \\ \text { BRT } & 89 & 84 & 77 & 71 & 62 & 55\end{array}$

Number of Patients At Risk

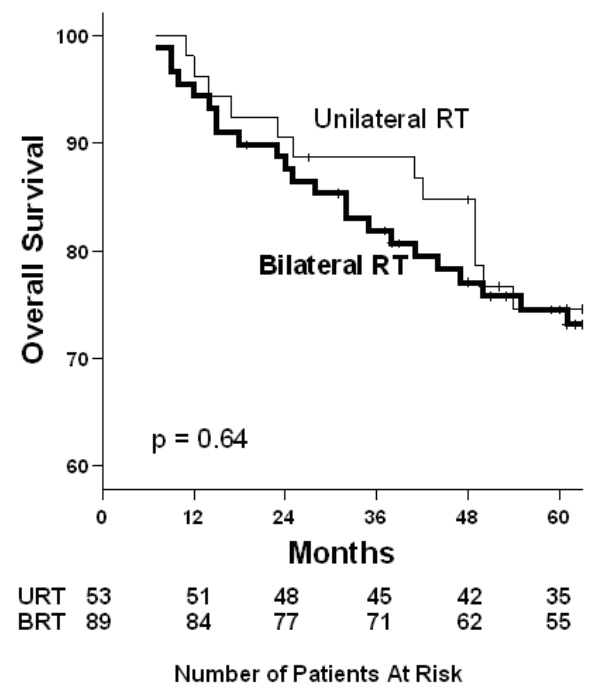

FIGURE 3: Outcomes for p16-positive subjects, by laterality of RT 


\section{Discussion}

We, and others, have previously published evidence that supports the practice of ipsilateral radiation therapy for selected cases of tonsillar SCC [15-16, 19-21]. In a broader context, there is also evidence to support this practice in patients treated with primary surgery, and not just amongst tonsillar primaries [22]. Although there is a variability in the stage of cancers included, the radiotherapy series all report rates of CLR less than 5\%. We utilised similar criteria for the selection of URT but saw a higher proportion of more advanced nodal disease in our population. However, overall CLR rates in this series remain comparable, at $7.1 \%$ for URT and $0.9 \%$ for BRT. While these results are statistically different, they remain low overall, and less than $10 \%$ (which has previously been accepted as a reasonable threshold for elective neck irradiation) [23].

In the modern era with increasing use of altered fractionation, concurrent chemotherapy, and increasingly conformal radiotherapy techniques, outcomes have improved from five-year DSS of approximately $60 \%$ in the earlier series to $75-80 \%$ in the more recent publications.

With increasing rates of HPV-associated cancer and the accompanying presentation of more advanced nodal disease [4-5], the question is whether we are treating a new disease which would require a shift in the treatment approach, namely, routine bilateral neck irradiation. In this series, amongst the p16-positive subjects, the risk of developing contralateral recurrence remained low overall (3.5\%) and was very similar to the rates seen in p16-negative subjects (2.5\%). While URT was associated with higher rates of CLR in p16-positive subjects (7.5\%) than with BRT (1.1\%), long-term outcomes were not affected, as LC, LRC, DFS, DSS, and OS remained similar between the two radiation techniques. This is likely due to both overall low rates of CLR and the efficacy of salvage treatment. Three of the four contralateral recurrences that developed in patients after unilateral radiation were effectively treated with a neck dissection, which provided control of the neck disease.

If we are to accept a slightly higher rate of CLR at the cost of a higher rate of salvage neck dissection, it is important to note the morbidity associated with this combination of treatment modalities. The late effects associated with neck radiation have been well-described to include xerostomia - which causes an increased risk of dental caries, neck fibrosis, dysphagia, and cerebrovascular events [10-12, 14, 24]. With salvage surgery, risks such as delayed healing, wound infection, or major bleeding must be considered. In general, these risks are estimated to be two to three times higher post-irradiation [24]. The most important long-term morbidity after neck dissection is shoulder dysfunction. This usually takes the form of shoulder pain and impairments in strength and range of motion [25]. While there is a lack of comparative evidence, it is felt that neck dissections for contralateral nodal recurrences can be done with little morbidity [26].

The American College of Radiology has published guidelines on how to select patients with tonsil SCC for URT. In it, nodal stage greater or equal to N2b is considered criteria for BRT [27]. However, in our data when examining the outcomes by tumour and nodal stage, it is reassuring that there is no significant difference in CLR rates. While more advanced $\mathrm{T}$ stage trended towards increased CLR rates, this was not true for advanced $\mathrm{N}$ stage. In fact, in our series, none of the p16-positive subjects with N2b who were treated with URT developed contralateral recurrences. In a small series from Delaware, patients with node-positive tonsil cancer, up to and including N2b disease, were treated with URT. Many (70\%) were treated after surgery, including neck dissection. No contralateral nodal failures were observed, leading the authors to conclude that the extent of the primary tumour is a more important predictor of failure than the nodal status [28]. Two other series lend support to our findings. Twenty patients from a Korean series treated unilaterally had T1-T3, N0-N2a disease. The primary tumours did not cross the midline and had less than $1 \mathrm{~cm}$ of tumour invasion into the soft palate or base of 
tongue (BOT). With a median follow-up of 64 months, the five-year local progression-free survival, distant metastasis-free survival, and progression-free survival rates were $95 \%, 100 \%$, and 95\%, respectively. The exclusion of extensive palate or BOT invasion was felt to be a possible reason for the excellent disease outcomes [29]. Furthermore, commentary and data from Peter MacCallum Cancer Centre details subjects with T1-4, N0-2b or N3 were treated with URT and none of the patients experienced contralateral nodal failure [21, 30].

In the above series, PET staging was widely used. In our older cohort, only twelve (6.5\%) subjects underwent staging PET evaluation prior to treatment, leading to a plausible risk of nodal under-staging. In today's era of routine PET imaging and, therefore, increased staging accuracy, the possibility of delivering radiotherapy to a patient who harbours contralateral nodal disease is presumably lower. In fact, El Khodary, et al, have shown that PET-CT yields additional diagnostic information in just under half of HNSCC patients and can modify the radiotherapy plan in up to a quarter [31].

To the best of our knowledge, this is the first series to review outcomes of URT by HPV status. A recent SEER analysis attempted to address this question by examining the trends associated with tumour size and nodal presentation [17]. The data demonstrated an increase in advanced regional disease in all tumour stages, but not necessarily an increase in bilateral nodal disease. Those authors concluded that there is no justification for altering the current practice of URT for well-lateralised disease. The results of our series support their conclusion.

While intrinsic differences between HPV-positive and HPV-negative head and neck squamous cell carcinoma (HNSCC) is not the focus of this paper, it is of interest that, despite similar rates of disease control, p16-positive subjects were shown to have superior OS. This is likely related to the differences in patient characteristics between the two groups, with p16-positive subjects tending to be younger (fewer comorbidities) and less likely to be smokers (lower risk of other malignancies).

\section{Conclusions}

Our findings confirm the relatively good prognosis associated with p16-positive squamous cell carcinomas of the tonsil compared to malignancies that are p16-negative. Overall, the rate of contralateral recurrence following unilateral radiation therapy was low, and there was no difference in the rates of disease control and overall survival when compared to patients who received bilateral treatment. Furthermore, even when considering only p16-positive subjects, unilateral versus bilateral radiation therapy did not alter disease-free or overall survival outcomes. While advanced $\mathrm{T}$ stage appeared to trend with increased rates of contralateral recurrence, no such association was seen for advanced nodal disease. Despite the increased burden of nodal disease associated with p16 positivity, we believe that unilateral radiation therapy in well-lateralised squamous cell carcinoma of the tonsil remains a safe and effective treatment. These conclusions are within the context of a retrospective review with small numbers of subjects with very advanced nodal disease.

\section{Additional Information}

\section{Disclosures}

Human subjects: Consent was obtained by all participants in this study. UBC British Columbia Cancer Agency REB issued approval H11-00364. Animal subjects: All authors have confirmed that this study did not involve animal subjects or tissue. Conflicts of interest: In compliance with the ICMJE uniform disclosure form, all authors declare the following: Payment/services info: All authors have declared that no financial support was received from any organization for the submitted work. Financial relationships: All authors have declared that they have no 
financial relationships at present or within the previous three years with any organizations that might have an interest in the submitted work. Other relationships: All authors have declared that there are no other relationships or activities that could appear to have influenced the submitted work.

\section{Acknowledgements}

The authors gratefully acknowledge the invaluable assistance of Dr. Tom Thompson, Dr. Catherine Poh, and Y. Kelly Liu in the determination of the p16 status of all pathological samples.

\section{References}

1. Psyrri A, Gouveris P, Vermorken JB: Human papillomavirus-related head and neck tumors: clinical and research implication. Curr Opin Oncol. 2009, 21:201-205.

10.1097/CCO.0b013e328329ab64

2. Auluck A, Hislop G, Bajdik C, Poh C, Zhang L, Rosin M: Trends in oropharyngeal and oral cavity cancer incidence of human papillomavirus (HPV)-related and HPV-unrelated sites in a multicultural population: the British Columbia experience. Cancer. 2010, 116:2635-2644. $10.1002 /$ cncr.25087

3. Chaturvedi AK, Engels EA, Anderson WF, Gillison ML: Incidence trends for human papillomavirus-related and -unrelated oral squamous cell carcinomas in the United States. J Clin Oncol. 2008, 26:612-619. 10.1200/JCO.2007.14.1713

4. Lassen P: The role of human papillomavirus in head and neck cancer and the impact on radiotherapy outcome. Radiother Oncol. 2010, 95:371-380. 10.1016/j.radonc.2010.04.022

5. Shonka DC Jr, Shoushtari AN, Thomas CY, Moskaluk C, Read PW, Reibel JF, Levine PA, Jameson MJ: Predicting residual neck disease in patients with oropharyngeal squamous cell carcinoma treated with radiation therapy: utility of p16 status. Arch Otolaryngol Head Neck Surg. 2009, 135:1126-1132. 10.1001/archoto.2009.153

6. Fakhry C, Westra WH, Li S, Cmelak A, Ridge JA, Pinto H, Forastiere A, Gillison ML: Improved survival of patients with human papillomavirus-positive head and neck squamous cell carcinoma in a prospective clinical trial. J Natl Cancer Inst. 2008, 100:261-269. 10.1093/jnci/djn011

7. Lassen P, Eriksen JG, Hamilton-Dutoit S, Tramm T, Alsner J, Overgaard J: Effect of HPVassociated p16INK4A expression on response to radiotherapy and survival in squamous cell carcinoma of the head and neck. J Clin Oncol. 2009, 27:1992-1998. 10.1200/JCO.2008.20.2853

8. Licitra L, Perrone F, Bossi P, Suardi S, Mariani L, Artusi R, Oggionni M, Rossini C, Cantù G, Squadrelli M, Quattrone P, Locati LD, Bergamini C, Olmi P, Pierotti MA, Pilotti S: High-risk human papillomavirus affects prognosis in patients with surgically treated oropharyngeal squamous cell carcinoma. J Clin Oncol. 2006, 24:5630-5636. 10.1200/JCO.2005.04.6136

9. Shoushtari A, Meeneghan M, Sheng K, Moskaluk CA, Thomas CY, Reibel JF, Levine PA, Jameson MJ, Keene K, Read PW: Intensity-modulated radiotherapy outcomes for oropharyngeal squamous cell carcinoma patients stratified by p16 status. Cancer. 2010, 116:2645-2654. 10.1002/cncr.25040

10. Henson BS, Eisbruch A, D'Hondt E, Ship JA: Two-year longitudinal study of parotid salivary flow rates in head and neck cancer patients receiving unilateral neck parotid-sparing radiotherapy treatment. Oral Oncol. 1999, 35:234-241. 10.1016/S1368-8375(98)00104-3

11. Leslie MD, Dische S: The early changes in salivary gland function during and after radiotherapy given for head and neck cancer. Radiother Oncol. 1994, 30:26-32. 10.1016/01678140(94)90006-X

12. Jensen K, Overgaard M, Grau C: Morbidity after ipsilateral radiotherapy for oropharyngeal cancer. Radiother Oncol. 2007, 85:90-97. 10.1016/j.radonc.2007.06.005

13. Wang ZH1, Yan C, Zhang ZY, Zhang CP, Hu HS, Tu WY, Kirwan J, Mendenhall WM: Impact of salivary gland dosimetry on post-IMRT recovery of saliva output and xerostomia grade for head-and-neck cancer patients treated with or without contralateral submandibular gland sparing: a longitudinal study. Int J Radiat Oncol Biol Phys. 2011, 81:1479-1487.

10.1016/j.ijrobp.2010.07.1990 
14. Scott AS, Parr LA, Johnstone PA: Risk of cerebrovascular events after neck and supraclavicular radiotherapy: a systematic review. Radiother Oncol. 2009, 90:163-165.

10.1016/j.radonc.2008.12.019

15. Jackson SM, Hay JH, Flores AD, Weir L, Wong FL, Schwindt C, Baerg B: Cancer of the tonsil: the results of ipsilateral radiation treatment. Radiother Oncol. 1999, 51:123-128.

10.1016/S0167-8140(99)00051-1

16. O'Sullivan B, Warde P, Grice B, Goh C, Payne D, Liu FF, Waldron J, Bayley A, Irish J, Gullane P, Cummings B: The benefits and pitfalls of ipsilateral radiotherapy in carcinoma of the tonsillar region. Int J Radiat Oncol Biol Phys. 2001, 51:332-343. 10.1016/S03603016(01)01613-3

17. Galloway TJ, Lango MN, Burtness B, Mehra R, Ruth K, Ridge JA: Unilateral neck therapy in the human papillomavirus ERA: accepted regional spread patterns. Head Neck. 2013, 35:160-164. 10.1002/hed.22929

18. Shoushtari AN, Meeneghan M, Treharne GC, Reibel JF, Levine PA, Moskaluk CA, Jameson MJ, Read PW: Clinical nodal staging of T1-2 tonsillar squamous cell carcinoma stratified by p16 status and implications for ipsilateral neck irradiation. Cancer J. 2010, 16:284-287. 10.1097/PPO.0b013e3181ddd088

19. Al-Mamgani A, van Rooij P, Fransen D, Levendag P: Unilateral neck irradiation for welllateralized oropharyngeal cancer. Radiother Oncol. 2013, 106:69-73. 10.1016/j.radonc.2012.12.006

20. Chronowski GM, Garden AS, Morrison WH, Frank SJ, Schwartz DL, Shah SJ, Beadle BM, Gunn GB, Kupferman ME, Ang KK, Rosenthal DI: Unilateral radiotherapy for the treatment of tonsil cancer. Int J Radiat Oncol Biol Phys. 2012, 83:204-209. 10.1016/j.ijrobp.2011.06.1975

21. Liu C, Dutu G, Peters LJ, Rischin D, Corry J: Tonsillar cancer: The Peter MacCallum experience with unilateral and bilateral irradiation. Head Neck. 2014, 36:317-322. 10.1002/hed.23297

22. Vergeer MR, Doornaert PA, Jonkman A, Kaanders JH, van den Ende PL, de Jong MA, Leemans CR, Slotman BJ, Langendijk JA: Ipsilateral irradiation for oral and oropharyngeal carcinoma treated with primary surgery and postoperative radiotherapy. Int J Radiat Oncol Biol Phys. 2010, 78:682-688. 10.1016/j.ijrobp.2009.08.042

23. Marks JE, Devineni VR, Harvey J, Sessions DG: The risk of contralateral lymphatic metastases for cancers of the larynx and pharynx. Am J Otolaryngol. 1992, 13:34-39. 10.1016/01960709(92)90095-B

24. Trotti A: Toxicity in head and neck cancer: a review of trends and issues . Int J Radiat Oncol Biol Phys. 2000, 47:1-12. 10.1016/S0360-3016(99)00558-1

25. Laverick S, Lowe D, Brown JS, Vaughan ED, Rogers SN: The impact of neck dissection on health-related quality of life. Arch Otolaryngol Head Neck Surg. 2004, 130:149-154. 10.1001/archotol.130.2.149

26. Cerezo L, Martín M, López M, Marín A, Gómez A: Ipsilateral irradiation for well lateralized carcinomas of the oral cavity and oropharynx: results on tumor control and xerostomia. Radiat Oncol. 2009, 4:33. 10.1186/1748-717X-4-33

27. Expert Panel on Radiation Oncology--Head \& Neck Cancer:, Yeung AR, Garg MK, Lawson J, McDonald MW, Quon H, Ridge JA, Saba N, Salama JK, Smith RV, Yom SS, Beitler JJ; American College of Radiology: ACR Appropriateness Criteria ${ }^{\circledR}$ ipsilateral radiation for squamous cell carcinoma of the tonsil. Head Neck. 2012, 34:613-616. 10.1002/hed.21993

28. Rusthoven KE, Raben D, Schneider C, Witt R, Sammons S, Raben A: Freedom from local and regional failure of contralateral neck with ipsilateral neck radiotherapy for node-positive tonsil cancer: results of a prospective management approach. Int J Radiat Oncol Biol Phys. 2009, 74:1365-1370. 10.1016/j.ijrobp.2008.10.023

29. Koo TR, Wu HG: Long-term results of ipsilateral radiotherapy for tonsil cancer . Radiat Oncol J. 2013, 31:66-71. 10.3857/roj.2013.31.2.66

30. Liu C, Corry J, Peters L: Letter to the editor regarding ACR Appropriateness Criteria for ipsilateral radiation for squamous cell carcinoma of the tonsil. Head Neck. 2013, 35:464. 10.1002/hed.23207

31. El-Khodarya M, Tabashyb R, Omarc W, Mousad A, Mostafa A: The role of PET/CT in the management of head and neck squamous cell carcinoma. The Egyptian Journal of Radiology and Nuclear Medicine. 2011, 42:157-167. 10.1016/j.ejrnm.2011.05.006 